\title{
Panorama de la maladie de Kaposi dans le service des maladies infectieuses et Tropicales du CHU Yalgado Ouédraogo de 2010 à 2015.
}

\author{
Panorama of Kaposi's sarcoma in the Department of Infectious and Tropical Diseases \\ of the Yalgado Ouédraogo University Hospital from 2010 to 2015
}

\author{
SAVADOGO $\mathrm{M}^{1}$, ZOUNGRANA $\mathrm{J}^{2}$, Boushab $\mathrm{MB}^{5}$, SONDO K A ${ }^{1}$, DIALLO I ${ }^{3}$, BAMBARA H A ${ }^{4}$, KYELEM \\ $\mathrm{N}^{1}$
}

1 service des maladies infectieuses du CHU Yalgado Ouédraogo

2 service des maladies infectieuses du CHU Sourou Sanon

3 service de médecine interne du CHU Yalgado Ouédraogo

4 service d'oncologie du CHU Yalgado Ouédraogo

5 Service de Médecine interne du Centre Hospitalier d'Aïoun, Mauritanie

Auteur correspondant : Savadogo Mamoudou Maître assistant à l'UFR/SDS université de Ouagadougou Burkina Faso Médecin infectiologue au CHU Yalgado Ouédraogo Email : savadoma@gmail.com

\begin{abstract}
Résumé
L'incidence de la maladie de Kaposi à l'ère de la multithérapie aux antirétroviraux est en croissance. Elle se manifeste couramment par des lésions papulo-nodulaires angiomateuses se localisant fréquemment au niveau des membres inférieurs. Sa gravité réside dans ses possibilités de dissémination locorégionale. A partir de cinq cas, les auteurs rappellent la diversité clinique de la maladie, la difficulté de confirmation du diagnostic dans notre contexte, et la gravité de certaines formes cliniques. La maladie concernait trois hommes et deux femmes dont l'âge variait entre 30 et 43 ans. Il s'agissait de trois cas de Kaposi cutané, un cas de kaposi œsophagien et un cas de Kaposi ulcéro bourgeonnante de jambe avec atteinte pleurale. La confirmation anatomopathologique a été faite chez un seul patient et deux cas étaient décédés en cours d'hospitalisation. La maladie de Kaposi était la circonstance de dépistage de l'infection à VIH dans deux cas, elle est survenue au cours du traitement antirétroviral dans trois cas. La maladie de Kaposi est une pathologie classant Sida. Son diagnostic chez le sujet vivant avec le VIH justifie l'instauration rapide d'un traitement antirétroviral efficace quel que soit la valeur des lymphocytes TCD4. Sa prise en charge requiert une collaboration pluridisciplinaire.
\end{abstract}

Mots clés : Maladie de Kaposi, VIH, Sida.

\begin{abstract}
The incidence of Kaposi's sarcoma in the era of Highly Active Antiretroviral Therapy (HAART) is growing. It is commonly manifesting by nodular-papular angiomatous lesions frequently located in the lower limbs. Its severity lies in its potential for loco-regional spread. From five cases, the authors recall the clinical diversity of the disease, the difficulty to confirm the diagnosis in our context and the severity of some clinical forms. Three men and two women between 30 and 43 years old had the disease. There were three cases of cutaneous Kaposi's sarcoma, one case of esophageal Kaposi's sarcoma and one case of sarcoma ulcerative budding leg with pleural affection. The histopathological confirmation was made in only one patient and two cases had died during hospitalization. Kaposi's sarcoma disease was the circumstance of HIV infection screening in two cases; it occurred during antiretroviral therapy in three cases. The Kaposi sarcoma is an AIDS classifying disease. Its diagnosis in subjects with HIV justifies the rapid introduction of effective antiretroviral therapy regardless of CD4 lymphocyte value. Its management requires a multidisciplinary collaboration.
\end{abstract}

Keywords: Kaposi’s sarcoma, HIV, AIDS.

\section{Introduction}

La maladie de Kaposi (SK) est un processus prolifératif mésenchymateux concernant les cellules des systèmes sanguin et lymphatique (5). Elle est décrite pour la première fois en 1872 par un dermatologue hongrois Moritz Kaposi sous le terme de Sarcoma idiopathicum multiplex pigmentosum (5). C'est une des affections opportunistes les plus fréquentes et le premier cancer des personnes vivant avec le virus de l'immunodéficience humaine (VIH). Elle est liée à l'infection par l'herpès virus humain 8 (HHV8)(19). En Occident la prévalence de la Maladie de Kaposi est moindre qu'en Afrique $(9,10,11,12)$. Traoré et coll au Burkina Faso retrouvait une prévalence de 80,6\% dans le service de dermatologie, tandis que Kodio au Mali retrouvaient une prévalence moindre $(13 \%)$ dans le service de médecine interne $(14,15)$. L'épidémie de SIDA a provoqué une explosion du nombre de 
cas de sarcomes de Kaposi, notamment dans les régions d'Afrique où l'HHV8 existe de façon endémique. $\mathrm{Au}$ service des maladies infectieuses du CHU YO, aucune étude ne s'est intéressée à cette pathologie. C'est pourquoi nous avons mené cette étude avec pour objectif de déterminer le profil clinique et les facteurs associés à cette pathologie dans le service des maladies infectieuses.

\section{Patients et méthode}

Cadre de l'étude

Le service des maladies infectieuses est un des services chargé de la prise en charge de l'infection à VIH et des affections opportunistes.

Type d'étude et critères d'inclusion

Il s'agit d'une étude rétrospective à visée descriptive qui s'est déroulée de 2010 à 2015.

Etaient inclus les patients hospitalisés ou suivis en ambulatoire au service des Maladies infectieuses, chez qui le diagnostic de la maladie de Kaposi a été posé par la clinique et/ou à l'anatomopathologie. Un avis d'un dermatologue a été nécessaire pour tous les cas cutanés.

\section{Observations}

Cas 1: Patient de 43 ans, dépisté le 23/09/2010 VIH1 positif, suite au décès de son épouse avec un taux de CD4 a 147/ml. L'initiation du traitement antirétroviral(TAR) a été faite le 20/10/2010 avec AZT+3TC+EFV. L'évolution sous TAR a été marquée par l'apparition de nodules violacées au niveau de la cheville gauche qui s'est accompagné progressivement d'une tuméfaction dure de la jambe homolatérale avant la première année de TAR évoquant la maladie de Kaposi(cf image 1). Un bilan d'extension à la recherche de d'autres localisations surtout digestive et pulmonaire étaient sans particulier. Le patient n'avait pas les moyens pour la prise en charge médicale spécifique de la MK. Le patient est toujours suivi en ambulatoire et il y a une amelioration de ses lésions de Kaposi.

Cas 2: Patiente de 30 ans VIH1 positif et sous atripla depuis le 28/08/2013 avec une notion de mauvaise observance du traitement, est hospitalisée le 16/09/2013 pour AEG + vomissements post prandiaux incoercibles et odynophagie. L'examen clinique notait une anémie modérée, une déshydratation et une dénutrition. La biologie notait des signes d'insuffisance rénale avec une créatininémie à $196 \mathrm{micromol} / \mathrm{l}$ et l'échographie rénale objectivait une souffrance rénale stade II. La fibroscopie digestive haute demandée, a noté une œsophagite peptique grade III en amont d'une hernie hiatale de petit volume. La biopsie réalisée grâce à la fibroscopie digestive haute, a permis à l'examen anatomopathologique de conclure à la Maladie Kaposi. Elle est décédée dans le service. (la reponse de l'anatomopathologie était: les lésions cadre bien avec la maladie de Kaposi sans autres précision)
Cas 3: Patiente de 34 ans, veuve depuis quatre années, VIH 1 positif non encore sous ARV a été admise le 6/02/2014 pour fièvre, vomissement, ictère, diarrhée et lésions cutanées. L'examen de la peau et des phanères montrait des lésions maculo papuleuses grossièrement ovalaires de taille variable allant de 0,2 à $1 \mathrm{~cm}$ de grand axe, d'allure angiomateuse évoquant une maladie de Kaposi disséminée sur tout le corps. La goutte épaisse était positive et des lésions évoquant un herpès génital étaient notées. Elle est classée au stade IV OMS. Elle est décédée dans un tableau de choc septique avant initiation du traitement antirétroviral.

Cas 4: Patient de 38 ans, référé au CHU YO pour hyperthermie vespérale, et asthénie. A l'admission au service des maladies infectieuses une anémie clinique, une conscience claire, des discrets œdèmes des membres inférieurs prenant le godet, un souffle systolique d'intensité $2 / 6$ d'allure fonctionnel, une température à $37^{\circ} 5$, un pouls à 80 bat $/ \mathrm{mn}$, une tension artérielle à $100 / 50 \mathrm{mmHg}$, une splénomégalie type I, une adénite suppurée axillaire droit. L'hémogramme notait une anémie sévère à $4,7 \mathrm{~g} / \mathrm{dl}$, la créatininémie élevée à 182 micromol/l (IR fonctionnelle), la SRV était positive, le taux de lymphocyte TCD4 à 138 cell/mm3. Le diagnostic de myocardiopathie anémique compliquée d'une insuffisance rénale fonctionnelle sur infection à VIH de découverte récente a été retenu. Sous traitement, l'évolution a été favorable au bout de 14 jours d'hospitalisation. $\mathrm{Au}$ troisième mois du TAR, le dosage des lymphocytes TCD4 est revenu à $300 / \mathrm{mm}^{3}$ et l'examen de la peau notait des lésions cutanées de Kaposi évoquant un syndrome de restauration immunitaire(cf image2). Le patient est toujours suivi en ambulatoire aussi bien dans le service des maladies infectieuses que dans le service de dermatologie.

Cas 5: Patient de 40 ans référé au CHU YO le 25 février 2015, pour ulcère associé à une tuméfaction douloureuse de la jambe gauche qui évoluait depuis 6 mois chez un patient dépisté VIH1 positif de découverte récente. L'examen notait à son admission des lésions ulcéro-bourgeonnantes suintante, purulente, saignant au contact et mesurant 18 à $20 \mathrm{~cm}$ siégeant à la jambe gauche. L'examen notait par ailleurs de multiples lésions nodulaires sur un important œdème qui s'étend sur tout l'hémicorps homolatéral et aux organes génitaux externes. L'examen de l'appareil respiratoire notait une polypnée à 32 cycles/mn, des signes d'épanchement pulmonaire gauche, confirmés à la radiographie pulmonaire. L'examen $\mathrm{du}$ système splénoganglionnaire relevait des adénopathies aux aires ganglionnaires superficielles. Devant ce tableau suspect de sarcome de Kaposi ulcéro bourgeonnante compliqué de pleurésie, une ponction pleurale a ramené un liquide hématique dont l'analyse était en 
faveur d'un transsudat. Le bilan de retentissement a été demandé et comportait le dosage des lymphocytes TCD4 $=591 / \mathrm{mm}^{3}$, la numération notait une anémie à $8,2 \mathrm{~g} / \mathrm{dl}$, la créatininémie $=52 \mathrm{micromol} / \mathrm{l}$, les $\mathrm{ALAT}=8 \mathrm{U} / \mathrm{l}$, la glycémie $=8,5 \mathrm{mmol} / 1$. Sous traitement, l'évolution était favorable et il est sorti de l'hôpital le 3/04/2015.

\section{Discussion}

La maladie concernait trois hommes et deux femmes dont l'âge variait entre 30 et 43 ans, comparable à ce qui est rapporté par plusieurs auteurs $(1,3)$. De même la prédominance masculine de la maladie avait déjà été rapportée dans la littérature avec un risque estimé à 14 fois plus élevé chez l'homme que chez la femme. La majorité des cas avait une atteinte cutanée qui est la forme dominante $(2,7)$. Un cas de Kaposi digestif avait été noté. Mais la fréquence de l'atteinte digestive est moindre (2). La maladie était survenue dans le cadre d'un syndrome de restauration immunitaire chez un patient. Sur le plan physiopathologique, il a été démontré que l'IRIS était associé à la restauration d'une réponse immune très inflammatoire dirigée contre l'agent pathogèneinfectieux présent $(1,4)$. Rattachée aux sarcomes ou bien aux angiomes, placée entre les tumeurs malignes et les processus bénins, décrite initialement dans certaines populations méditerranéennes puis en Afrique, cette maladie est restée longtemps énigmatique (5). En 1981 au Center for Diseases Control d'Atlanta, FriedmanKien rapportait une épidémie de sarcome de Kaposi (SK) disséminé, associé à une pneumopathie à Pneumocystis jirovecii chez des homosexuels immunodéprimés (1). Peu après le virus de l'immunodéficience humaine (VIH) est identifié et l'hypothèse infectieuse du Sarcome de Kaposi est relancée. Plus tard Chang établissait l'origine virale de la maladie (6). Tous nos patients étaient des adultes jeunes et infectés par le VIH, comme relevé par Traoré au Burkina Faso et Kodio au Mali (14). En effet le risque de développer un sarcome de Kaposi lors du sida est estimé à 20000 fois celui de la population générale et près de 300 fois celui des autres sujets immunodéprimés $(8,15)$. Une thérapie antivirale spécifique du sarcome de Kaposi n'est cependant pas encore établie. La progression du sarcome peut toutefois être stoppée sous un traitement antirétroviral puissant, les lésions reculent même fréquemment $(17,18)$. En présence d'une affection étendue surtout viscérale, il faut envisager une chimiothérapie oncologique (16).

\section{Conclusion}

La maladie de Kaposi est une pathologie peu fréquente au service des Maladies infectieuses à l'ère de la multi thérapie antirétrovirale. L'aspect clinique est polymorphe mais dominée par les atteintes cutanées avec une association fréquente de comorbidité. Les formes disséminées et les formes digestives étaient les plus graves. Sa prise en charge requiert une collaboration pluridisciplinaire. Un dépistage précoce et un bon suivi des patients infectés par le VIH permettront de prévenir les infections opportunistes graves comme la maladie de Kaposi.

\section{Références}

1) Chakib A., Hliwa W., Marih L., Himmich $H$. Maladie de Kaposi au cours de l'infection par le VIH au Maroc. (À propos de 50 cas). Bull Soc Pathol Exot, 2003, 96, 2, 86-89

2) Kadjo $\mathrm{K}$ et coll. Endoscopie digestive haute et maladie de kaposi. Médecine d'Afrique Noire : 1998, 45 (5)

3) Christeff N, Winter C, Gharakhanian S, Thobie N, Costagliola D. Differences in androgens of HIV positive patients with and without Kaposi's sarcoma. Journal of Clinical Pathology, 1995; 48 (6): 513-518.

4) Silvia Sovaila, Claire Rivoisy, Anne Bourgarit. Le syndrome de restauration immunitaire: plusieurs visages pour le même syndrome (p.8-18), Médecine thérapeutique - Volume 21, numéro 1, Janvier-Février 2015

5) Morand J.J., Lightburn E., Simon F., Patte J.H. Actualités de la maladie de kaposi. Med Trop 2007 ; 67 : 123-130

6) Chang Y, Cesarman E, Pessinms et Coll Identification of herpesvirus+like DNA sequences in AIDS associated Kaposi's sarcoma. Science 1994 ; 266 : 1865-69.

7) Ngaroua1, Mbo Amvene J, Neossi N M, Eloundou N J, Masso M P, Essomba A. Sarcome de Kaposi Gastrique à Sérologie VIH Négative : à Propos d'un Cas à l'Hôpital Régional de Ngaoundéré. Health Sci. Dis: Vol 14 (1) March 2013

8) Aubry P. (2007) Maladie de Kaposi. Médecine Tropicale. Actualité 2007

9) Garait V; Molina JM. Infection par le VIH. Rev Prat 2000; $60: 1003-10$

10) Guillot B, Meynadier J. Maladie de Kaposi. Encycl Med chir. (Dermatologie paris, France 12790 A, 4-1986 : $8 p$.

11) Plancoulaine S, Gessain A. Aspects épidémiologiques de l'herpesvirus humain 8 (HHV-8) et du sarcome de Kaposi. Med Mal infect, 2005, 35 (5) :314-321.

12) Chakib A, Hlima W, Marih L, Himmich $H$. Maladie de Kaposi au cours de l'infection par le VIH au Maroc. Bull Soc Path Exot, 2003; 96: 8689.

13) Yassibanda S, Koffi B, Bobossi SG, Fossi M. Maladie de Kaposi à Bangui : aspect épidémiologique, anatomo-clinique et étiopathogénique, relation avec HHV8. Med Afr Noire, 2002; 49: 455-9.

14) Barro-Traoré Fatou, Traoré Adama, Konaté Issouf, Traoré Si Simon, Sawadogo Nobila Oumar, Sanou Issa, Soudré B. Robert, Heid Àric, Grosshans 
Àdouard. Tumeurs cutanéo-muqueuses : aspects épidémiologiques dans le service de dermatologie du centre hospitalier national Yalgado Ouédraogo de Ouagadougou, Burkina Faso Volume 13, numéro 2, Avril 2003.

15) Kodio B, Cissé I Ah, Konaré Hd, Dembélé M, Rhaly A. fréquence des consultations dermatologiques dans les activités du service de médecine interne de l'hôpital national du point $\mathrm{G}$ à Bamako. Mali médical 2014 TOME XXIX N³

16) H. Furrer. Traitement et prévention des infections opportunistes les plus importantes associées au virus HIV. Forum Med Suisse No 236 juin 2001

17) Bower M, Fox P, Fife K, Gill J, Nelson M, Gazzard B. Highly active anti-retroviral therapy (HAART) prolongs time to treatment failure in Kaposi's sarcoma. AIDS 1999;13: 2105-2111.

18) Krischer J, Rutschmann O, Hirschel B, Vollenweider-Roten S, Saurat JH, Pechere M. Regression of Kaposi's sarcoma during therapy with HIV-1 protease inhibitors: a prospective pilot study. J Am Acad Dermatol 1998;38:594-8.

19) Bender Ignacio RA, Goldman JD, Magaret AS , Selke S, Huang ML, Gantt S, Johnston C, Phipps WT, Schiffer JT, Zuckerman RA, McClelland RS, Celum C, Corey L, Wald A, Casper C. Patterns of human herpesvirus- 8 oral shedding among diverse cohorts of human herpesvirus- 8 seropositive persons. Infect Agent Cancer. 2016 Feb 10;11:7. doi: 10.1186/s13027-016-0052-2. eCollection 2016

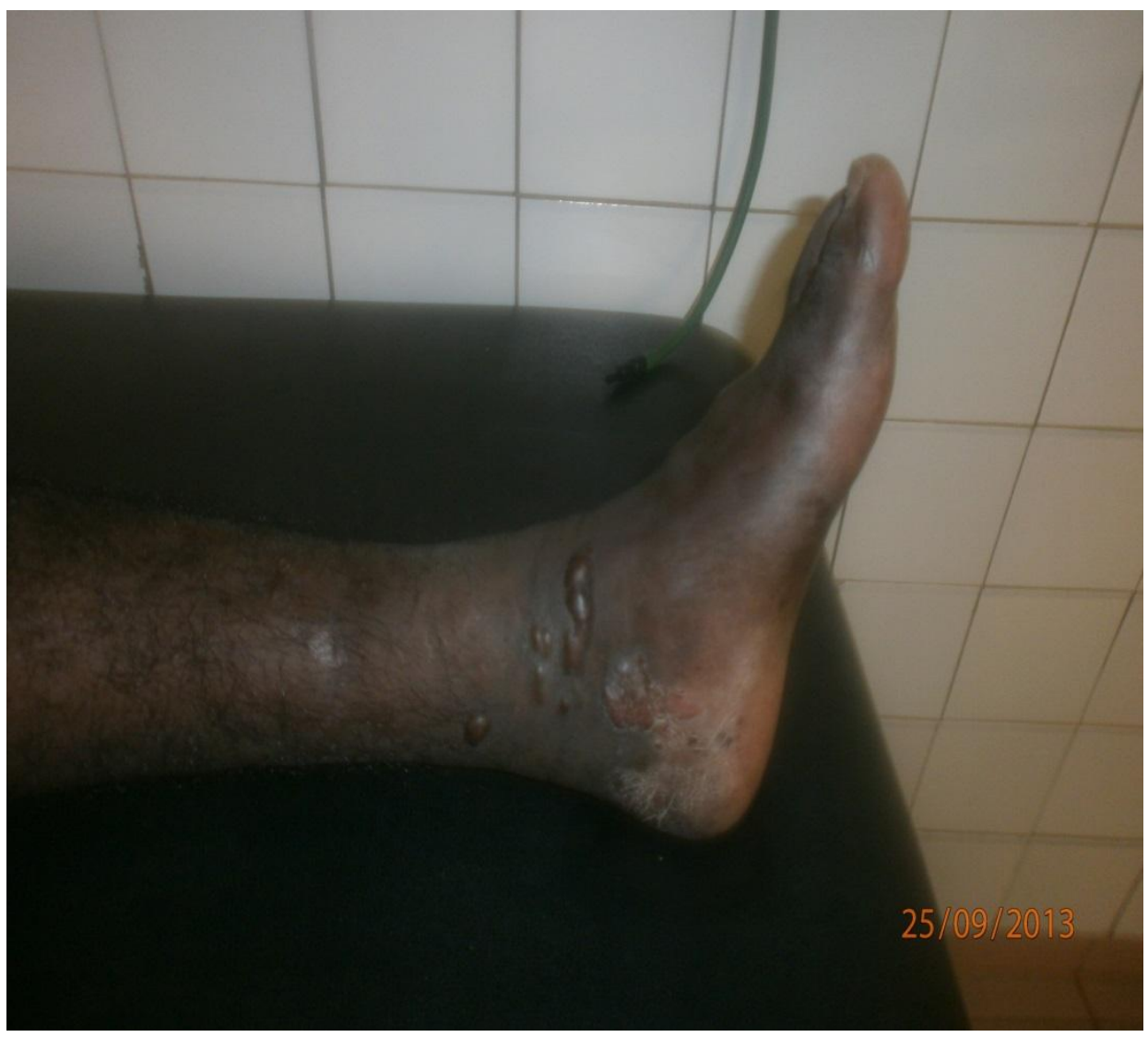

Image1 : de kaposi jambe 


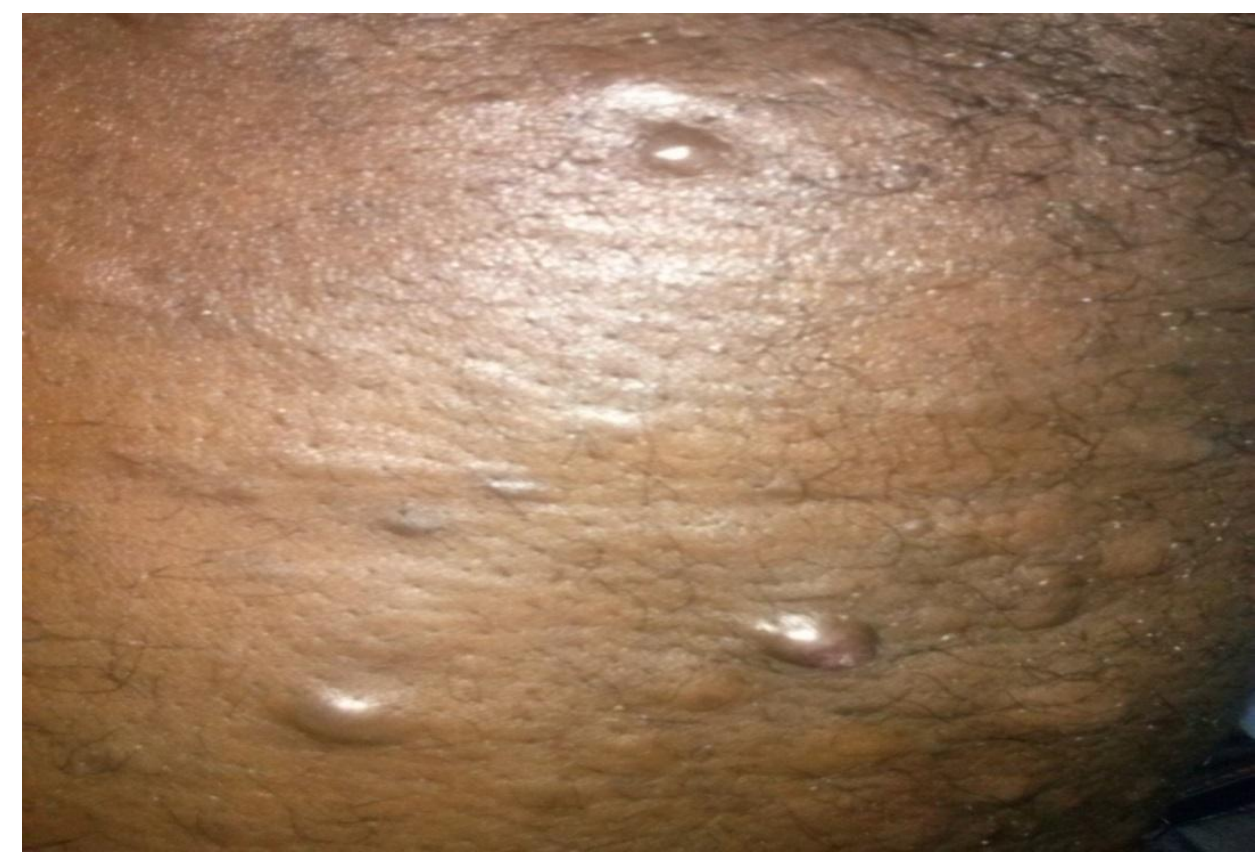

Image2 : de Kaposi crurale infiltrée avec lymphœdème 